\title{
(Regular) pseudo-bosons versus bosons
}

\author{
F. Bagarello \\ Dipartimento di Metodi e Modelli Matematici, Facoltà di Ingegneria, \\ Università di Palermo, I-90128 Palermo, Italy \\ e-mail: bagarell@unipa.it \\ Home page: www.unipa.it/ bagarell
}

\begin{abstract}
We discuss in which sense the so-called regular pseudo-bosons, recently introduced by Trifonov and analyzed in some details by the author, are related to ordinary bosons. We repeat the same analysis also for pseudo-bosons, and we analyze the role played by certain intertwining operators, which may be bounded or not.
\end{abstract}




\section{Introduction}

In a series of recent papers 1, 2, 3, 4, we have investigated some mathematical aspects of the so-called pseudo-bosons (PB), originally introduced by Trifonov in [5]. They arise from the canonical commutation relation $\left[a, a^{\dagger}\right]=\mathbb{1}$ upon replacing $a^{\dagger}$ by another (unbounded) operator $b$ not (in general) related to $a:[a, b]=\mathbb{1}$. We have shown that, under suitable assumptions, $N=b a$ and $\mathfrak{N}=N^{\dagger}=a^{\dagger} b^{\dagger}$ can be both diagonalized, and that their spectra coincide with the set of natural numbers (including 0 ), $\mathbb{N}_{0}$. However the sets of related eigenvectors are not orthonormal (o.n.) bases but, nevertheless, they are automatically biorthogonal. In most of the examples considered so far, they are bases of the Hilbert space of the system, $\mathcal{H}$, and, in some cases, they turn out to be Riesz bases.

In [6] and [7] some physical examples arising from concrete models in quantum mechanics have been discussed. These examples suggested to introduce the difference between regular pseudo-bosons (RPB) and PB: the RPB, see Section II, arise when the two sets of eigenvectors of $N$ and $\mathfrak{N}$ are mapped one into the other by a bounded operator with bounded inverse. If this operator is unbounded, then we have to do with PB. PB have also been considered by other authors recently, see [8] for instance, without calling them in this way. These PB have been shown to have to do with the so-called pseudo-hermitian quantum mechanics, which in recent years have became more and more appealing since it considers the possibility of having non self-adjoint hamiltonians with real spectra, showing that this possibility is related to some commutativity conditions between the hamiltonian itself and the parity and the time reversal operators, [9]. The same feature, more from a mathematical side, has been analyzed for instance in [10, 11. Of course, these references should be considered just as a starting point for a deeper analysis.

In this paper we consider the relation between $\mathrm{PB}, \mathrm{RPB}$, and ordinary bosons, proving two similar theorems, one for $\mathrm{PB}$ and the other for RPB. More in details: in the next section we introduce and discuss some features of $d$-dimensional PB. In Sections III we prove our main theorem for RPB, while Section IV contains an analogous result for PB, together with some

physical examples; we will see that techniques of unbounded operators are the natural tools in that case. We give our conclusions in Section V. 


\section{II $d$-dimensional $\mathrm{PB}$ and RPB}

In this section we will construct a $d$-dimensional $(d$-D) version of what originally proposed in [1], to which we refer for further comments on the 1-D situation.

Let $\mathcal{H}$ be a given Hilbert space with scalar product $\langle.,$.$\rangle and related norm \|$.$\| . We intro-$ duce $d$ pairs of operators, $a_{j}$ and $b_{j}, j=1,2, \ldots, d$, acting on $\mathcal{H}$ and satisfying the following commutation rules

$$
\left[a_{j}, b_{j}\right]=\mathbb{1}
$$

where $j=1,2, \ldots, d$, all the other commutators being trivial. Of course, they collapse to the CCR's for $d$ independent modes if $b_{j}=a_{j}^{\dagger}, j=1,2, \ldots, d$. It is well known that $a_{j}$ and $b_{j}$ are unbounded operators, so they cannot be defined on all of $\mathcal{H}$. Following [1], and writing $D^{\infty}(X):=\cap_{p \geq 0} D\left(X^{p}\right)$ (the common domain of all the powers of the operator $X$ ), we consider the following:

Assumption 1.- there exists a non-zero $\varphi_{\mathbf{0}} \in \mathcal{H}$ such that $a_{j} \varphi_{\mathbf{0}}=0, j=1,2, \ldots, d$, and $\varphi_{\mathbf{0}} \in D^{\infty}\left(b_{1}\right) \cap D^{\infty}\left(b_{2}\right) \cap \cdots \cap D^{\infty}\left(b_{d}\right)$.

Assumption 2.- there exists a non-zero $\Psi_{\mathbf{0}} \in \mathcal{H}$ such that $b_{j}^{\dagger} \Psi_{\mathbf{0}}=0, j=1,2, \ldots, d$, and $\Psi_{0} \in D^{\infty}\left(a_{1}^{\dagger}\right) \cap D^{\infty}\left(a_{2}^{\dagger}\right) \cap \cdots \cap D^{\infty}\left(a_{d}^{\dagger}\right)$.

Under these assumptions we can introduce the following vectors in $\mathcal{H}$ :

$$
\left\{\begin{array}{l}
\varphi_{\mathbf{n}}:=\varphi_{n_{1}, n_{2}, \ldots, n_{d}}=\frac{1}{\sqrt{n_{1} ! n_{2} ! \cdots n_{d} !}} b_{1}^{n_{1}} b_{2}^{n_{2}} \cdots b_{d}^{n_{d}} \varphi_{\mathbf{0}} \\
\Psi_{\mathbf{n}}:=\Psi_{n_{1}, n_{2}, \ldots, n_{d}}=\frac{1}{\sqrt{n_{1} ! n_{2} ! \cdots n_{d} !}} a_{1}^{\dagger n_{1}} a_{2}^{\dagger n_{2}} \cdots a_{d}^{\dagger n_{d}} \Psi_{\mathbf{0}}
\end{array}\right.
$$

$n_{j}=0,1,2, \ldots$, for all $j=1,2, \ldots, d$. Let us now define the unbounded operators $N_{j}:=b_{j} a_{j}$ and $\mathfrak{N}_{j}:=N_{j}^{\dagger}=a_{j}^{\dagger} b_{j}^{\dagger}, j=1,2, \ldots, d$. It is possible to check that $\varphi_{\mathbf{n}}$ belongs to the domain of $N_{j}, D\left(N_{j}\right)$, and that $\Psi_{\mathbf{n}} \in D\left(\mathfrak{N}_{j}\right)$, for all possible $\mathbf{n}$. Moreover,

$$
N_{j} \varphi_{\mathbf{n}}=n_{j} \varphi_{\mathbf{n}}, \quad \mathfrak{N}_{j} \Psi_{\mathbf{n}}=n_{j} \Psi_{\mathbf{n}}
$$

Under the above assumptions, and if we chose the normalization of $\Psi_{\mathbf{0}}$ and $\varphi_{\mathbf{0}}$ in such a way that $\left\langle\Psi_{\mathbf{0}}, \varphi_{\mathbf{0}}\right\rangle=1$, we find that

$$
\left\langle\Psi_{\mathbf{n}}, \varphi_{\mathbf{m}}\right\rangle=\delta_{\mathbf{n}, \mathbf{m}}=\prod_{j=1}^{d} \delta_{n_{j}, m_{j}}
$$

This means that the sets $\mathcal{F}_{\Psi}=\left\{\Psi_{\mathbf{n}}\right\}$ and $\mathcal{F}_{\varphi}=\left\{\varphi_{\mathbf{n}}\right\}$ are biorthogonal and, because of this, the vectors of each set are linearly independent. If we now call $\mathcal{D}_{\varphi}$ and $\mathcal{D}_{\Psi}$ respectively the linear 
span of $\mathcal{F}_{\varphi}$ and $\mathcal{F}_{\Psi}$, and $\mathcal{H}_{\varphi}$ and $\mathcal{H}_{\Psi}$ their closures, then

$$
f=\sum_{\mathbf{n}}\left\langle\Psi_{\mathbf{n}}, f\right\rangle \varphi_{\mathbf{n}}, \quad \forall f \in \mathcal{H}_{\varphi}, \quad h=\sum_{\mathbf{n}}\left\langle\varphi_{\mathbf{n}}, h\right\rangle \Psi_{\mathbf{n}}, \quad \forall h \in \mathcal{H}_{\Psi} .
$$

What is not in general ensured is that the Hilbert spaces introduced so far all coincide, i.e. that $\mathcal{H}_{\varphi}=\mathcal{H}_{\Psi}=\mathcal{H}$. Indeed, we can only state that $\mathcal{H}_{\varphi} \subseteq \mathcal{H}$ and $\mathcal{H}_{\Psi} \subseteq \mathcal{H}$. However, motivated by the examples discussed in the literature, we make the

Assumption 3.- The above Hilbert spaces all coincide: $\mathcal{H}_{\varphi}=\mathcal{H}_{\Psi}=\mathcal{H}$,

which was introduced in [1]. This means, in particular, that both $\mathcal{F}_{\varphi}$ and $\mathcal{F}_{\Psi}$ are bases of $\mathcal{H}$, so that the following resolutions of the identity, written in bra-ket notation, hold:

$$
\sum_{\mathbf{n}}\left|\varphi_{\mathbf{n}}\right\rangle\left\langle\Psi_{\mathbf{n}}\left|=\sum_{\mathbf{n}}\right| \Psi_{\mathbf{n}}\right\rangle\left\langle\varphi_{\mathbf{n}}\right|=\mathbb{1}
$$

Let us now introduce the operators $S_{\varphi}$ and $S_{\Psi}$ via their action respectively on $\mathcal{F}_{\Psi}$ and $\mathcal{F}_{\varphi}$ :

$$
S_{\varphi} \Psi_{\mathbf{n}}=\varphi_{\mathbf{n}}, \quad S_{\Psi} \varphi_{\mathbf{n}}=\Psi_{\mathbf{n}},
$$

for all $\mathbf{n}$, which in particular imply that $\Psi_{\mathbf{n}}=\left(S_{\Psi} S_{\varphi}\right) \Psi_{\mathbf{n}}$ and $\varphi_{\mathbf{n}}=\left(S_{\varphi} S_{\Psi}\right) \varphi_{\mathbf{n}}$, for all $\mathbf{n}$. Hence

$$
S_{\Psi} S_{\varphi}=S_{\varphi} S_{\Psi}=\mathbb{1} \quad \Rightarrow \quad S_{\Psi}=S_{\varphi}^{-1} .
$$

In other words, both $S_{\Psi}$ and $S_{\varphi}$ are invertible and one is the inverse of the other. Furthermore, we can also check that they are both positive, well defined and symmetric, [1. Moreover, at least formally, it is possible to write these operators as

$$
S_{\varphi}=\sum_{\mathbf{n}}\left|\varphi_{\mathbf{n}}><\varphi_{\mathbf{n}}\right|, \quad S_{\Psi}=\sum_{\mathbf{n}}\left|\Psi_{\mathbf{n}}><\Psi_{\mathbf{n}}\right| .
$$

These expressions are only formal, at this stage, since the series may or may not converge in the uniform topology and the operators $S_{\varphi}$ and $S_{\Psi}$ could be unbounded. Indeed we know, [12], that two biorthogonal bases are related by a bounded operator, with bounded inverse, if and only if they are Riesz bases 1 . This is why in [1] we have also considered

Assumption 4. $-\mathcal{F}_{\varphi}$ and $\mathcal{F}_{\Psi}$ are Bessel sequences. In other words, there exist two positive constants $A_{\varphi}, A_{\Psi}>0$ such that, for all $f \in \mathcal{H}$,

$$
\sum_{\mathbf{n}}\left|\left\langle\varphi_{\mathbf{n}}, f\right\rangle\right|^{2} \leq A_{\varphi}\|f\|^{2}, \quad \sum_{\mathbf{n}}\left|\left\langle\Psi_{\mathbf{n}}, f\right\rangle\right|^{2} \leq A_{\Psi}\|f\|^{2} .
$$

\footnotetext{
${ }^{1}$ Recall that a set of vectors $\phi_{1}, \phi_{2}, \phi_{3}, \ldots$, is a Riesz basis of a Hilbert space $\mathcal{H}$, if there exists a bounded operator $V$, with bounded inverse, on $\mathcal{H}$, and an orthonormal basis of $\mathcal{H}, \varphi_{1}, \varphi_{2}, \varphi_{3}, \ldots$, such that $\phi_{j}=V \varphi_{j}$, for all $j=1,2,3, \ldots$
} 
This assumption is equivalent to require that $\mathcal{F}_{\varphi}$ and $\mathcal{F}_{\Psi}$ are both Riesz bases, and implies that $S_{\varphi}$ and $S_{\Psi}$ are bounded operators: $\left\|S_{\varphi}\right\| \leq A_{\varphi},\left\|S_{\Psi}\right\| \leq A_{\Psi}$. Moreover $\frac{1}{A_{\Psi}} \mathbb{1} \leq S_{\varphi} \leq A_{\varphi} \mathbb{1}$, and $\frac{1}{A_{\varphi}} \mathbb{1} \leq S_{\Psi} \leq A_{\Psi} \mathbb{1}$. Hence the domains of $S_{\varphi}$ and $S_{\Psi}$ can be taken to be all of $\mathcal{H}$. While Assumptions 1, 2 and 3 are quite often satisfied, as the examples contained in our previous papers and in the recent review [13] show, it is quite difficult to find physical examples satisfying also Assumption 4. On the other hand, it is rather easy to find mathematical examples satisfying all the assumptions, see Section II.1 below. Hence, as announced, we introduce the following difference: we call pseudo-bosons (PB) those excitations satisfying the first three assumptions, while, if Assumption 4 is also satisfied, these will be called regular pseudo-bosons (RPB). Clearly, RPB are PB, but the converse is false, in general.

Generalizing what already discussed in [1, 7], these $d$-dimensional pseudo-bosons give rise to interesting intertwining relations among non self-adjoint operators, see also [3] and references therein. In particular it is easy to check that

$$
S_{\Psi} N_{j}=\mathfrak{N}_{j} S_{\Psi} \quad \text { and } \quad N_{j} S_{\varphi}=S_{\varphi} \mathfrak{N}_{j}
$$

$j=1,2, \ldots, d$. This is related to the fact that the spectra of, say, $N_{1}$ and $\mathfrak{N}_{1}$ coincide and that their eigenvectors are related by the operators $S_{\varphi}$ and $S_{\Psi}$, see equations (2.3) and (2.7), in agreement with the literature on intertwining operators, [14, 15], and on pseudo-Hermitian quantum mechanics, see [9, 10, 11] and references therein.

\section{II.1 Construction of RPB}

We will show here that each Riesz basis produces some RPB. Let $\mathcal{F}_{\varphi}:=\left\{\varphi_{\mathbf{n}}\right\}$ be a Riesz basis of $\mathcal{H}$ with bounds $A$ and $B, 0<A \leq B<\infty$. The associated frame operator $S:=\sum_{\mathbf{n}}\left|\varphi_{\mathbf{n}}><\varphi_{\mathbf{n}}\right|$ is bounded, positive and admits a bounded inverse. Also, the set $\mathcal{F}_{\hat{\varphi}}:=\left\{\hat{\varphi}_{\mathbf{n}}:=S^{-1 / 2} \varphi_{\mathbf{n}}\right\}$ is an o.n. basis of $\mathcal{H}$. Therefore we can define $d$ lowering operators $a_{j, \hat{\varphi}}$ on $\mathcal{F}_{\hat{\varphi}}$ as $a_{j, \hat{\varphi}} \hat{\varphi}_{\mathbf{n}}=\sqrt{n_{j}} \hat{\varphi}_{\mathbf{n}_{\mathbf{j}-}}$, and their adjoints, $a_{j, \hat{\varphi}}^{\dagger}$, as $a_{j, \hat{\varphi}}^{\dagger} \hat{\varphi}_{\mathbf{n}}=\sqrt{n_{j}+1} \hat{\varphi}_{\mathbf{n}_{\mathbf{j}+}}$. Here $\mathbf{n}_{j-}=\left(n_{1}, \ldots, n_{j}-1, \ldots, n_{d}\right)$ and $\mathbf{n}_{j+}=\left(n_{1}, \ldots, n_{j}+1, \ldots, n_{d}\right)$. Hence $\left[a_{j, \hat{\varphi}}, a_{k, \hat{\varphi}}^{\dagger}\right]=\delta_{j, k} \mathbb{1}$. If we now define $a_{j}:=S^{1 / 2} a_{j, \hat{\varphi}} S^{-1 / 2}$, this acts on the Riesz basis $\mathcal{F}_{\varphi}$ as a lowering operator. However, since $\mathcal{F}_{\varphi}$ is not an o.n. basis in general, $a_{j}^{\dagger}$ is not a raising operator, so that $\left[a_{j}, a_{k}^{\dagger}\right] \neq \delta_{j, k} \mathbb{1}$. However, if we now define the operator $b_{j}:=S^{1 / 2} a_{j, \hat{\varphi}}^{\dagger} S^{-1 / 2}$, it is clear that in general $b_{j} \neq a_{j}^{\dagger}$, and $b_{j}$ acts on $\varphi_{\mathbf{n}}$ as

a raising operator: $b_{j} \varphi_{\mathbf{n}}=\sqrt{n_{j}+1} \varphi_{\mathbf{n}_{j+}}$, for all $\mathbf{n}$. Then we have $\left[a_{j}, b_{k}\right]=\delta_{j, k} \mathbb{1}$. So we have constructed two sets of operators satisfying (2.1) and which are not related by a simple conjugation. This is not the end of the story. Indeed: 
1. Assumption 1 is verified since $\varphi_{\mathbf{0}}$ is annihilated by $a_{j}$ and belongs to the domain of all the powers of $b_{j}$.

2. As for Assumption 2, it is enough to define $\Psi_{\mathbf{0}}=S^{-1} \varphi_{\mathbf{0}}$. With this definition $b_{j}^{\dagger} \Psi_{\mathbf{0}}=0$ and $\Psi_{\mathbf{0}}$ belongs to the domain of all the powers of $a_{j}^{\dagger}$.

3. Since $\mathcal{F}_{\varphi}$ is a Riesz basis of $\mathcal{H}$ by assumption, then $\mathcal{H}_{\varphi}=\mathcal{H}$. Notice now that the vectors $\Psi_{\mathbf{n}}$ can be written as $\Psi_{\mathbf{n}}=S^{-1} \varphi_{\mathbf{n}}$, for all $\mathbf{n}$. Hence $\mathcal{F}_{\Psi}$ is in duality with $\mathcal{F}_{\varphi}$ and therefore is a Riesz basis of $\mathcal{H}$ as well. Hence $\mathcal{H}_{\Psi}=\mathcal{H}$. This proves Assumption 3.

4. As for Assumption 4, this is equivalent to the hypothesis originally assumed here, i.e. that $\mathcal{F}_{\varphi}$ is a Riesz basis.

Explicit examples arising from this general construction can be found in [4].

\section{II.2 Coherent states}

As it is well known there exist several different, and not always equivalent, ways to define coherent states, [16, 17]. In this paper, following [1], we will adopt the following definition: let $z_{j}, j=1,2, \ldots, d$ be $d$ complex variables, $z_{j} \in \mathcal{D}$ (some domain in $\mathbb{C}$ ), and let us introduce the following operators:

$$
\left\{\begin{array}{l}
U_{j}\left(z_{j}\right)=e^{z_{j} b_{j}-\bar{z}_{j} a_{j}}=e^{-\left|z_{j}\right|^{2} / 2} e^{z_{j} b_{j}} e^{-\bar{z}_{j} a_{j}}, \\
V_{j}\left(z_{j}\right)=e^{z_{j} a_{j}^{\dagger}-\bar{z}_{j} b_{j}^{\dagger}}=e^{-\left|z_{j}\right|^{2} / 2} e^{z_{j} a_{j}^{\dagger}} e^{-\bar{z}_{j} b_{j}^{\dagger}}
\end{array}\right.
$$

$j=1,2, \ldots, d$

$$
\left\{\begin{array}{l}
U\left(z_{1}, z_{2}, \ldots, z_{d}\right):=U_{1}\left(z_{1}\right) U_{2}\left(z_{2}\right) \cdots U_{d}\left(z_{d}\right), \\
V\left(z_{1}, z_{2}, \ldots, z_{d}\right):=V_{1}\left(z_{1}\right) V_{2}\left(z_{2}\right) \cdots V_{d}\left(z_{d}\right),
\end{array}\right.
$$

and the following vectors:

$$
\varphi\left(z_{1}, z_{2}, \ldots, z_{d}\right)=U\left(z_{1}, z_{2}, \ldots, z_{d}\right) \varphi_{\mathbf{0}}, \quad \Psi\left(z_{1}, z_{2}, \ldots, z_{d}\right)=V\left(z_{1}, z_{2}, \ldots, z_{d}\right) \Psi_{\mathbf{0}}
$$

Remarks:- (1) Due to the commutation rules for the operators $b_{j}$ and $a_{j}$, we clearly have $\left[U_{j}\left(z_{j}\right), U_{k}\left(z_{k}\right)\right]=\left[V_{j}\left(z_{j}\right), V_{k}\left(z_{k}\right)\right]=0$, for $j \neq k$.

(2) Since the operators $U$ and $V$ are, for generic $z_{j}$, unbounded, definition (2.14) makes sense only if $\varphi_{\mathbf{0}} \in D(U)$ and $\Psi_{\mathbf{0}} \in D(V)$, a condition which will be assumed here. In [1] it was proven that, for instance, this is so when $\mathcal{F}_{\varphi}$ and $\mathcal{F}_{\Psi}$ are Riesz bases.

(3) The set $\mathcal{D}$ could be, in principle, a proper subset of $\mathbb{C}$. 
It is possible to write the vectors $\varphi\left(z_{1}, z_{2}, \ldots, z_{d}\right)$ and $\Psi\left(z_{1}, z_{2}, \ldots, z_{d}\right)$ in terms of the vectors of $\mathcal{F}_{\Psi}$ and $\mathcal{F}_{\varphi}$ as

$$
\left\{\begin{array}{l}
\varphi\left(z_{1}, z_{2}, \ldots, z_{d}\right)=e^{-\left(\left|z_{1}\right|^{2}+\left|z_{2}\right|^{2}+\ldots+\left|z_{d}\right|^{2}\right) / 2} \sum_{\mathbf{n}} \frac{z_{1}^{n_{1}} z_{2}^{n_{2} \ldots z_{d}}}{\sqrt{n_{1} ! n_{2} ! \ldots n_{d} !}} \varphi_{\mathbf{n}} \\
\Psi\left(z_{1}, z_{2}, \ldots, z_{d}\right)=e^{-\left(\left|z_{1}\right|^{2}+\left|z_{2}\right|^{2}+\ldots+\left|z_{d}\right|^{2}\right) / 2} \sum_{\mathbf{n}} \frac{z_{1}^{n_{1}} z_{2}^{n_{2} \ldots z_{d}}}{\sqrt{n_{1} ! n_{2} ! \ldots n_{d} !}} \Psi_{\mathbf{n}}
\end{array}\right.
$$

These vectors are called coherent since they are eigenstates of the lowering operators. Indeed we can check that

$$
a_{j} \varphi\left(z_{1}, z_{2}, \ldots, z_{d}\right)=z_{j} \varphi\left(z_{1}, z_{2}, \ldots, z_{d}\right), \quad b_{j}^{\dagger} \Psi\left(z_{1}, z_{2}, \ldots, z_{d}\right)=z_{j} \Psi\left(z_{1}, z_{2}, \ldots, z_{d}\right),
$$

for $j=1,2, \ldots, d$ and $z_{j} \in \mathcal{D}$. It is also a standard exercise, putting $z_{j}=r_{j} e^{i \theta_{j}}$, to check that the following operator equalities hold:

$$
\left\{\begin{array}{l}
\frac{1}{\pi^{d}} \int_{\mathbb{C}} d z_{1} \int_{\mathbb{C}} d z_{2} \ldots \int_{\mathbb{C}} d z_{d}\left|\varphi\left(z_{1}, z_{2}, \ldots, z_{d}\right)><\varphi\left(z_{1}, z_{2}, \ldots, z_{d}\right)\right|=S_{\varphi} \\
\frac{1}{\pi^{d}} \int_{\mathbb{C}} d z_{1} \int_{\mathbb{C}} d z_{2} \ldots \int_{\mathbb{C}} d z_{d}\left|\Psi\left(z_{1}, z_{2}, \ldots, z_{d}\right)><\Psi\left(z_{1}, z_{2}, \ldots, z_{d}\right)\right|=S_{\Psi}
\end{array}\right.
$$

as well as

$$
\frac{1}{\pi^{d}} \int_{\mathbb{C}} d z_{1} \int_{\mathbb{C}} d z_{2} \ldots \int_{\mathbb{C}} d z_{d}\left|\varphi\left(z_{1}, z_{2}, \ldots, z_{d}\right)><\Psi\left(z_{1}, z_{2}, \ldots, z_{d}\right)\right|=\sum_{\mathbf{n}}\left|\varphi_{\mathbf{n}}\right\rangle\left\langle\Psi_{\mathbf{n}}\right|=\mathbb{1},
$$

which are written in convenient bra-ket notation. It should be said that these equalities are, most of the times, only formal results. Indeed, extending an analogous result given in [7] for $d=2$, we can prove the following

Theorem 1 Let $a_{j}, b_{j}, \mathcal{F}_{\varphi}, \mathcal{F}_{\Psi}, \varphi\left(z_{1}, z_{2}, \ldots, z_{d}\right)$ and $\Psi\left(z_{1}, z_{2}, \ldots, z_{d}\right)$ be as above. Let us assume that (1) $\mathcal{F}_{\varphi}, \mathcal{F}_{\Psi}$ are Riesz bases; (2) $\mathcal{F}_{\varphi}, \mathcal{F}_{\Psi}$ are biorthogonal. Then (2.18) holds true.

Suppose therefore that the above construction gives coherent states that do not satisfy a resolution of the identity (see [2] for an example). Then, since $\mathcal{F}_{\varphi}$ and $\mathcal{F}_{\Psi}$ are automatically biorthogonal, they cannot be Riesz bases (neither one of them)!

\section{RPB versus bosons}

In this section we will prove the following theorem, given in $d=1$ for simplicity, establishing a sort of equivalence between RPB and ordinary bosons. This equivalence is related to the 
existence of a bounded operator $T$ with bounded inverse and of a pair of conjugate operators $c$ and $c^{\dagger}$ satisfying the canonical commutation rule $\left[c, c^{\dagger}\right]=\mathbb{1}$, which are related with the original pair of operators $a$ and $b$. More in details we have:

Theorem 2 Let $a$ and $b$ be two operators on $\mathcal{H}$ satisfying $[a, b]=\mathbb{1}$, and for which Assumptions 1, 2, 3 and 4 of Section II are satisfied. Then an unbounded, densely defined, operator $c$ on $\mathcal{H}$ exists, together with a positive bounded operator $T$ with bounded inverse $T^{-1}$, such that $\left[c, c^{\dagger}\right]=\mathbb{1}$. Moreover

$$
a=T c T^{-1}, \quad b=T c^{\dagger} T^{-1} .
$$

Viceversa, given an unbounded, densely defined, operator $c$ on $\mathcal{H}$ satisfying $\left[c, c^{\dagger}\right]=\mathbb{1}$ and a positive bounded operator $T$ with bounded inverse $T^{-1}$, two operators a and $b$ can be introduced for which $[a, b]=\mathbb{1}$, and for which equations (3.1) and Assumptions 1, 2, 3 and 4 of Section II are satisfied.

\section{Proof -}

To prove the first part of the theorem we first remind that, because of Assumption 4 of Section II, the operators $S_{\varphi}$ and $S_{\Psi}$ defined as in (2.9),

$$
S_{\varphi} f=\sum_{n=0}^{\infty}\left\langle\varphi_{n}, f\right\rangle \varphi_{n}, \quad S_{\Psi} f=\sum_{n}\left\langle\Psi_{n}, f\right\rangle \Psi_{n},
$$

$f \in \mathcal{H}$, are well defined, bounded and positive (hence, self-adjoint). Also, $S_{\varphi}=S_{\Psi}^{-1}$. These are standard results in the theory of Riesz bases, [12, 18]. In particular, choosing the normalization constants in $\Psi_{0}$ and $\varphi_{0}$ in such a way that $\left\langle\Psi_{0}, \varphi_{0}\right\rangle=1$, we know that $\left\langle\Psi_{n}, \varphi_{m}\right\rangle=\delta_{n, m}$ and, as a consequence,

$$
S_{\varphi} \Psi_{m}=\varphi_{m}, \quad S_{\Psi} \varphi_{m}=\Psi_{m},
$$

for all $m \geq 0$. Because of the properties of $S_{\Psi}$ and $S_{\varphi}$, their square roots surely exist and, for instance, $S_{\varphi}^{-1 / 2}=S_{\Psi}^{1 / 2}$. Hence we define the vectors $\hat{\varphi}_{n}=S_{\varphi}^{-1 / 2} \varphi_{n}, n \geq 0$, and the related set $\mathcal{F}_{\hat{\varphi}}=\left\{\hat{\varphi}_{n}, n \geq 0\right\}$. It is well known that $\mathcal{F}_{\hat{\varphi}}$ is an o.n. basis of $\mathcal{H}$, and it coincides with the o.n. basis we would construct introducing (apparently) new vectors $\hat{\Psi}_{n}=S_{\Psi}^{-1 / 2} \Psi_{n}, n \geq 0$, since it can be easily checked that, for all $n, \hat{\Psi}_{n}=\hat{\varphi}_{n}$.

On $\mathcal{F}_{\hat{\varphi}}$ we can define the ordinary bosonic lowering and raising operators:

$$
\left\{\begin{array}{l}
c \hat{\varphi}_{n}=\sqrt{n} \hat{\varphi}_{n-1}, \\
c^{\dagger} \hat{\varphi}_{n}=\sqrt{n+1} \hat{\varphi}_{n+1}
\end{array}\right.
$$


with the convention that $c \hat{\varphi}_{0}=0$. Of course $\left[c, c^{\dagger}\right]=\mathbb{1}$. Recall now, [1], that our working hypotheses also imply that $a \varphi_{n}=\sqrt{n} \varphi_{n-1}$ and $b \varphi_{n}=\sqrt{n+1} \varphi_{n+1}$, which can be rewritten as $S_{\varphi}^{-1 / 2} a S_{\varphi}^{1 / 2} \hat{\varphi}_{n}=\sqrt{n} \hat{\varphi}_{n-1}$, and $S_{\varphi}^{-1 / 2} b S_{\varphi}^{1 / 2} \hat{\varphi}_{n}=\sqrt{n+1} \hat{\varphi}_{n+1}$. Hence $a, b$ and $c$ are related as follows:

$$
c=S_{\varphi}^{-1 / 2} a S_{\varphi}^{1 / 2}, \quad c^{\dagger}=S_{\varphi}^{-1 / 2} b S_{\varphi}^{1 / 2},
$$

which are exactly equations (3.1), identifying $T$ with $S_{\varphi}^{1 / 2}$.

Let us now prove the second part of the theorem. First of all, by means of $c$ and $c^{\dagger}$, we construct the o.n. basis $\mathcal{F}_{\hat{\varphi}}$ of $\mathcal{H}, \mathcal{F}_{\hat{\varphi}}=\left\{\hat{\varphi}_{n}=\frac{c^{t^{n}}}{\sqrt{n !}} \hat{\varphi}_{0}\right\}$, where $c \hat{\varphi}_{0}=0$. Then, since both $T$ and $T^{-1}$ are bounded and, therefore, everywhere defined, we can introduce two new families of vectors: $\mathcal{F}_{\varphi}=\left\{\varphi_{n}=T \hat{\varphi}_{n}, n \geq 0\right\}$ and $\mathcal{F}_{\Psi}=\left\{\Psi_{n}=T^{-1} \hat{\varphi}_{n}, n \geq 0\right\}$. These two families are obviously biorthogonal, $\left\langle\Psi_{n}, \varphi_{m}\right\rangle=\delta_{n, m}$, and they are both complete in $\mathcal{H}$ : so they are two (in general different) bases of $\mathcal{H}$. We can now define on, say, $\mathcal{F}_{\varphi}$, two operators $a$ and $b$ which act as lowering and raising operators:

$$
\left\{\begin{array}{l}
a \varphi_{n}=\sqrt{n} \varphi_{n-1}, \\
b \varphi_{n}=\sqrt{n+1} \varphi_{n+1},
\end{array}\right.
$$

for all $n \geq 0$. In particular the first equation implies that $a \varphi_{0}=0$. Incidentally we observe that $b^{\dagger} \neq a$, since $\mathcal{F}_{\varphi}$ is not, in general, an o.n. basis. Iterating the second equation in (3.5), we deduce that $\varphi_{n}=\frac{b^{n}}{\sqrt{n !}} \varphi_{0}$, which gives an alternative expression for the vector $\varphi_{n}$ and, moreover, shows that $\varphi_{0} \in D^{\infty}(b)$. Hence Assumption 1 is satisfied.

Since $\left(T c^{\dagger} T^{-1}\right) \varphi_{n}=T c^{\dagger} \hat{\varphi}_{n}=\sqrt{n+1} T \hat{\varphi}_{n+1}=\sqrt{n+1} \varphi_{n+1}$, and since $\mathcal{F}_{\varphi}$ is a basis of $\mathcal{H}$, we deduce that $b=T c^{\dagger} T^{-1}$. Analogously, we can prove that $a=T c T^{-1}$. It is now clear that $[a, b]=\mathbb{1}$ and that $a^{\dagger}=T^{-1} c^{\dagger} T$. To prove Assumption 2 we first notice that $b^{\dagger} \Psi_{0}=\left(T c^{\dagger} T^{-1}\right)^{\dagger}\left(T^{-1} \hat{\varphi}_{0}\right)=T^{-1} c \hat{\varphi}_{0}=0$. Moreover, since for all $n \geq 0$

$$
a^{\dagger} \Psi_{n}=\left(T^{-1} c^{\dagger} T\right) T^{-1} \hat{\varphi}_{n}=T^{-1} c^{\dagger} \hat{\varphi}_{n}=\sqrt{n+1} \Psi_{n+1},
$$

by iteration we deduce that $\Psi_{n}=\frac{a^{\dagger^{n}}}{\sqrt{n !}} \Psi_{0}$, which means that $\Psi_{0} \in D^{\infty}\left(a^{\dagger}\right)$. This prove Assumption 2, while Assumption 3 follows from our previous claim on $\mathcal{F}_{\varphi}$ and $\mathcal{F}_{\Psi}$ : they are both bases of $\mathcal{H}$. Finally, since they are obtained by the o.n. basis $\mathcal{F}_{\hat{\varphi}}$ by acting with the bounded operators $T$ or $T^{-1}$, they are also Riesz bases.

Remarks:- (1) The proof of the above theorem recall, at least in part, the construction given in Section II.1. This is not surprising since we are now dealing with Riesz bases. The difference will be evident in the next Section. 
(2) Theorem 2 implies that the intertwining operators in (2.11) for RPB are bounded, with bounded inverse.

\section{PB versus bosons}

In this section we will not assume that $T$ and $T^{-1}$ are bounded operators, and many domain problems will arise as a consequence. This will be related to the nature of the biorthogonal bases we work with, which will not be Riesz bases any longer. The relevance of this section, as widely explained in [13] and references therein, follows from the fact that all the physical examples seem to give rise to PB and not to RPB. From the mathematical side, we will formulate now a different theorem which is the analogue of the one proven in the previous section in this different settings and we will show that, even if part of that proof can be repeated here, most of the arguments should be changed to take care of unboundedness of the operators. As in the previous section, to simplify the proof and the notation, we fix $d=1$. Extension to $d>1$ is straightforward.

Theorem 3 Let $a$ and $b$ be two operators on $\mathcal{H}$ satisfying $[a, b]=\mathbb{1}$, and for which Assumptions 1, 2, and 3 (but not 4) of Section II are satisfied. Then two unbounded, densely defined, operators $c$ and $R$ on $\mathcal{H}$ exist, such that $\left[c, c^{\dagger}\right]=\mathbb{1}$ and $R$ is positive, self adjoint and admits an unbounded inverse $R^{-1}$. Moreover

$$
a=R c R^{-1}, \quad b=R c^{\dagger} R^{-1}
$$

and, introducing $\hat{\varphi}_{n}=\frac{c^{\dagger^{n}}}{\sqrt{n !}} \hat{\varphi}_{0}, c \varphi_{0}=0$, we have the following: $\hat{\varphi}_{n} \in D(R) \cap D\left(R^{-1}\right)$, for all $n \geq 0$, and the sets $\left\{R \hat{\varphi}_{n}\right\}$ and $\left\{R^{-1} \hat{\varphi}_{n}\right\}$ are biorthogonal bases of $\mathcal{H}$.

Viceversa, let us consider two unbounded, densely defined, operators $c$ and $R$ on $\mathcal{H}$ satisfying $\left[c, c^{\dagger}\right]=\mathbb{1}$ with $R$ positive, self-adjoint with unbounded inverse $R^{-1}$. Suppose that, introduced $\hat{\varphi}_{n}$ as above, $\hat{\varphi}_{n} \in D(R) \cap D\left(R^{-1}\right)$, for all $n \geq 0$, and that the sets $\left\{R \hat{\varphi}_{n}\right\}$ and $\left\{R^{-1} \hat{\varphi}_{n}\right\}$ are biorthogonal bases of $\mathcal{H}$. Then two operators $a$ and $b$ can be introduced for which $[a, b]=\mathbb{1}$, and for which equations (4.1) and Assumptions 1, 2, and 3 (but not 4) of Section II are satisfied.

\section{Proof -}

To prove the first part of the theorem we recall that the two sets $\mathcal{F}_{\varphi}=\left\{\varphi_{n}, n \geq 0\right\}$ and $\mathcal{F}_{\Psi}=\left\{\Psi_{n}, n \geq 0\right\}$ defined as in Section II are biorthogonal bases of $\mathcal{H}$ but they are not Riesz bases. Hence, defining

$$
S_{\varphi} \Psi_{n}=\varphi_{n}, \quad S_{\Psi} \varphi_{n}=\Psi_{n}
$$


for all $n \geq 0$, on the domains $D\left(S_{\varphi}\right)=$ linear $\operatorname{span}\left\{\Psi_{n}\right\}$ and $D\left(S_{\Psi}\right)=$ linear span $\left\{\varphi_{n}\right\}$, it follows from general results, [12, that both these operators are unbounded, so that they are not everywhere defined. It is possible to check that $\left\langle f, S_{\varphi} f\right\rangle \geq 0$ for all $f \in D\left(S_{\varphi}\right)$ and $\left\langle f, S_{\Psi} f\right\rangle \geq 0$ for all $f \in D\left(S_{\Psi}\right)$. In particular, if $f \neq 0$, both these mean values are strictly positive. It is straightforward to check that, as in the previous section, $S_{\varphi}=S_{\Psi}^{-1}$, and that both operators are symmetric:

$$
\begin{cases}\left\langle f, S_{\varphi} g\right\rangle=\left\langle S_{\varphi} f, g\right\rangle, & \forall f, g \in D\left(S_{\varphi}\right), \\ \left\langle f, S_{\Psi} g\right\rangle=\left\langle S_{\Psi} f, g\right\rangle, & \forall f, g \in D\left(S_{\Psi}\right) .\end{cases}
$$

In these conditions it is known, [19], that each one of these operators admits a self-adjoint extension, which is also positive. We call these extensions $\hat{S}_{\varphi}$ and $\hat{S}_{\Psi}$. Using standard results in functional calculus, we can now define square roots of these operators and the following holds:

$$
\hat{S}_{\varphi}=\hat{S}_{\Psi}^{-1}, \quad \hat{S}_{\varphi}^{1 / 2}=\hat{S}_{\Psi}^{-1 / 2}, \quad \hat{S}_{\varphi}^{-1 / 2}=\hat{S}_{\Psi}^{1 / 2}
$$

It is easy to check that, for all $n \geq 0, \varphi_{n} \in D\left(\hat{S}_{\varphi}^{-1 / 2}\right)$, so that $D\left(\hat{S}_{\Psi}\right)=D\left(\hat{S}_{\varphi}^{-1}\right) \subseteq D\left(\hat{S}_{\varphi}^{-1 / 2}\right)$. Indeed, we can check that $\left\|\hat{S}_{\varphi}^{-1 / 2} \varphi_{n}\right\|=1$. This is a particular case of the following more general result:

$$
\left\langle\hat{S}_{\varphi}^{-1 / 2} \varphi_{n}, \hat{S}_{\varphi}^{-1 / 2} \varphi_{k}\right\rangle=\left\langle\varphi_{n}, \hat{S}_{\varphi}^{-1} \varphi_{k}\right\rangle=\left\langle\varphi_{n}, \hat{S}_{\Psi} \varphi_{k}\right\rangle=\left\langle\varphi_{n}, \Psi_{k}\right\rangle=\delta_{n, k},
$$

due to the biorthogonality of $\mathcal{F}_{\varphi}$ and $\mathcal{F}_{\Psi}$. This suggests to introduce a third set of vectors of $\mathcal{H}, \mathcal{F}_{\hat{\varphi}}=\left\{\hat{\varphi}_{n}:=\hat{S}_{\varphi}^{-1 / 2} \varphi_{n}, n \geq 0\right\}$, which is made of o.n. vectors. As in Section III, defining $\hat{\Psi}_{n}=\hat{S}_{\Psi}^{-1 / 2} \Psi_{n}$, does not produce new vectors; again we get $\hat{\Psi}_{n}=\hat{\varphi}_{n} \forall n \geq 0$. We also deduce that $D\left(\hat{S}_{\varphi}\right) \subseteq D\left(\hat{S}_{\varphi}^{1 / 2}\right)$.

Let us notice that, since $D\left(\hat{S}_{\Psi}\right) \subseteq D\left(\hat{S}_{\varphi}^{-1 / 2}\right) \subset \mathcal{H}$ and since the closure of $D\left(\hat{S}_{\Psi}\right)$ returns $\mathcal{H}$, ${\overline{D\left(\hat{S}_{\varphi}^{-1 / 2}\right)}}^{\|\|}=\mathcal{H}$. Analogously, $\overline{D\left(\hat{S}_{\varphi}^{1 / 2}\right)} \|^{\|\|}=\mathcal{H}$. Moreover, $\forall n \geq 0, \hat{\varphi}_{n} \in D\left(\hat{S}_{\varphi}^{-1 / 2}\right) \cap D\left(\hat{S}_{\varphi}^{1 / 2}\right)$ : indeed, a straightforward computation shows that $\hat{S}_{\varphi}^{1 / 2} \hat{\varphi}_{n}=\varphi_{n}$ and that $\hat{S}_{\varphi}^{-1 / 2} \hat{\varphi}_{n}=\hat{S}_{\varphi}^{-1} \varphi_{n}=$ $\hat{S}_{\Psi} \varphi_{n}=\Psi_{n}$.

Finally, if $f \in D\left(\hat{S}_{\varphi}^{-1 / 2}\right)$ is orthogonal to all $\hat{\varphi}_{n}, f=0$. Hence, due to the density of $D\left(\hat{S}_{\varphi}^{-1 / 2}\right)$ in $\mathcal{H}$, we conclude that $\mathcal{F}_{\hat{\varphi}}$ is an o.n. basis of $\mathcal{H},\left[20\right.$. On $\mathcal{F}_{\hat{\varphi}}$ we define the standard annihilation operator $c$ as usual, $c \hat{\varphi}_{n}=\sqrt{n} \hat{\varphi}_{n-1}$, whose adjoint is the creation operator $c^{\dagger} \hat{\varphi}_{n}=\sqrt{n+1} \hat{\varphi}_{n+1}$. We can rewrite the first of these equation as $c \hat{S}_{\varphi}^{-1 / 2} \varphi_{n}=\sqrt{n} \hat{S}_{\varphi}^{-1 / 2} \varphi_{n-1}$, which implies, first of all, that $c \hat{S}_{\varphi}^{-1 / 2} \varphi_{n} \in D\left(\hat{S}_{\varphi}^{1 / 2}\right)$. Also, $\hat{S}_{\varphi}^{1 / 2} c \hat{S}_{\varphi}^{-1 / 2} \varphi_{n}=\sqrt{n} \varphi_{n-1}$ which, compared with $a \varphi_{n}=\sqrt{n} \varphi_{n-1}$, shows that $a=\hat{S}_{\varphi}^{1 / 2} c \hat{S}_{\varphi}^{-1 / 2}$.

In a similar way, $c^{\dagger} \hat{\varphi}_{n}=\sqrt{n+1} \hat{\varphi}_{n+1}$ can be rewritten as $c^{\dagger} \hat{S}_{\varphi}^{-1 / 2} \varphi_{n}=\sqrt{n+1} \hat{S}_{\varphi}^{-1 / 2} \varphi_{n-1}$. Therefore $c^{\dagger} \hat{S}_{\varphi}^{-1 / 2} \varphi_{n} \in D\left(\hat{S}_{\varphi}^{1 / 2}\right)$ and $\hat{S}_{\varphi}^{1 / 2} c^{\dagger} \hat{S}_{\varphi}^{-1 / 2} \varphi_{n}=\sqrt{n+1} \varphi_{n+1}$ which, compared with 
$b \varphi_{n}=\sqrt{n+1} \varphi_{n+1}$, shows that $b=\hat{S}_{\varphi}^{1 / 2} c^{\dagger} \hat{S}_{\varphi}^{-1 / 2}$. This proves (4.1), identifying $R$ with $\hat{S}_{\varphi}^{1 / 2}$. Also, since $R \hat{\varphi}_{n}=\hat{S}_{\varphi}^{1 / 2} \hat{\varphi}_{n}=\varphi_{n}$ and $R^{-1} \hat{\varphi}_{n}=\hat{S}_{\varphi}^{-1} \varphi_{n}=\Psi_{n}$, the linear spans of both $\left\{R \hat{\varphi}_{n}\right\}$ and $\left\{R^{-1} \hat{\varphi}_{n}\right\}$ are biorthogonal bases of $\mathcal{H}$.

Let us now prove the inverse statement. Because of our assumptions, the set $\mathcal{F}_{\hat{\varphi}}$ of vectors $\hat{\varphi}_{n}=\frac{c^{\dagger^{n}}}{\sqrt{n !}} \hat{\varphi}_{0}, c \varphi_{0}=0$, is an o.n. basis in $\mathcal{H}$ and $\hat{\varphi}_{n} \in D(R) \cap D\left(R^{-1}\right), \forall n \geq 0$. Then we define, for all $n \geq 0, \varphi_{n}=R \hat{\varphi}_{n}, \Psi_{n}=R^{-1} \hat{\varphi}_{n}, \mathcal{F}_{\varphi}=\left\{\varphi_{n}, n \geq 0\right\}, \mathcal{F}_{\Psi}=\left\{\Psi_{n}, n \geq 0\right\}$, and $D_{\varphi}$ and $D_{\Psi}$ their linear span, which are both dense in $\mathcal{H}$ since, by assumption, $\mathcal{F}_{\varphi}$ and $\mathcal{F}_{\Psi}$ are (biorthogonal) bases of $\mathcal{H}$.

We can now introduce lowering and raising operators on $\mathcal{F}_{\varphi}$ as in (3.5). In particular, iterating $b \varphi_{n}=\sqrt{n+1} \varphi_{n+1}$, we get $\varphi_{n}=\frac{b^{n}}{\sqrt{n !}} \varphi_{0}$ and we also find that $b^{\dagger} \Psi_{n}=\sqrt{n-1} \Psi_{n-1}$. The first equation, $a \varphi_{n}=\sqrt{n-1} \varphi_{n-1}$, produces $a^{\dagger} \Psi_{n}=\sqrt{n+1} \Psi_{n+1}$, which, again by iteration, gives $\Psi_{n}=\frac{a^{\dagger^{n}}}{\sqrt{n !}} \Psi_{0}$.

It is now a simple exercise to check that:

1. $a \varphi_{0}=0$ and $\varphi_{0} \in D^{\infty}(b)$. Hence Assumption 1 is satisfied.

2. $b^{\dagger} \Psi_{0}=0$ and $\Psi_{0} \in D^{\infty}\left(a^{\dagger}\right)$. Hence Assumption 2 is satisfied.

3. With similar techniques as in the first part of the proof we deduce that $b=R c R^{-1}$ and $a=R c R^{-1}$, which could also be checked computing directly their action on the vectors $\hat{\varphi}_{n}$.

4. ${\overline{D_{\varphi}}}^{\|\|}={\overline{D_{\Psi}}}^{\|\|}=\mathcal{H}$. Hence Assumption 3 is satisfied.

5. since $\mathcal{F}_{\varphi}$ and $\mathcal{F}_{\Psi}$ are obtained from the o.n. basis $\mathcal{F}_{\hat{\varphi}}$ via the action of an unbounded, invertible, operator with unbounded inverse, they cannot be Riesz bases, [12]. Hence Assumption 4 is violated.

This concludes the proof.

\section{IV.1 Physical examples}

We conclude this section with some examples, arising from quantum mechanics, in which the operators $\hat{S}_{\varphi}$ and $\hat{S}_{\Psi}$ can be explicitly identified. These examples are reviewed in [13, where the original references and more examples (even in $d>1$ ) can be found. 


\section{IV.1.1 The extended quantum harmonic oscillator}

The hamiltonian of this model, introduced in [21], is the non self-adjoint operator $H_{\beta}=$ $\frac{\beta}{2}\left(p^{2}+x^{2}\right)+i \sqrt{2} p$, where $\beta$ is a positive parameter and $[x, p]=i$. Introducing the standard bosonic operators $a=\frac{1}{\sqrt{2}}\left(x+\frac{d}{d x}\right), a^{\dagger}=\frac{1}{\sqrt{2}}\left(x-\frac{d}{d x}\right),\left[a, a^{\dagger}\right]=\mathbb{1}$, and the number operator $N=a^{\dagger} a$, we can write $H_{\beta}=\beta N+\left(a-a^{\dagger}\right)+\frac{\beta}{2} \mathbb{1}$ which, introducing further the operators

$$
\hat{A}_{\beta}=a-\frac{1}{\beta}, \quad \hat{B}_{\beta}=a^{\dagger}+\frac{1}{\beta},
$$

can be written as

$$
H_{\beta}=\beta\left(\hat{B}_{\beta} \hat{A}_{\beta}+\gamma_{\beta} \mathbb{1}\right),
$$

where $\gamma_{\beta}=\frac{2+\beta^{2}}{2 \beta^{2}}$. It is clear that, for all $\beta>0, \hat{A}_{\beta}^{\dagger} \neq \hat{B}_{\beta}$ and that $\left[\hat{A}_{\beta}, \hat{B}_{\beta}\right]=\mathbb{1}$. Hence we have to do with pseudo-bosonic operators which, as proved in [6], satisfy Assumptions 1, 2 and 3 but not Assumption 4. Indeed we have deduced that $\hat{S}_{\varphi}=e^{2\left(a+a^{\dagger}\right) / \beta}$, which is unbounded with unbounded inverse. We have PB which are not regular.

\section{IV.1.2 The Swanson hamiltonian}

The starting point is the following non self-adjoint hamiltonian, [21]:

$$
H_{\theta}=\frac{1}{2}\left(p^{2}+x^{2}\right)-\frac{i}{2} \tan (2 \theta)\left(p^{2}-x^{2}\right),
$$

where $\theta$ is a real parameter taking value in $\left(-\frac{\pi}{4}, \frac{\pi}{4}\right) \backslash\{0\}=: I$. It is clear that $H_{\theta}^{\dagger} \neq H_{\theta}$, for all $\theta \in I$. Introducing the annihilation and creation operators $a$ and $a^{\dagger}$ as usual, we write

$$
H_{\theta}=N+\frac{i}{2} \tan (2 \theta)\left(a^{2}+\left(a^{\dagger}\right)^{2}\right)+\frac{1}{2} \mathbb{1},
$$

where $N=a^{\dagger} a$. This hamiltonian can be still rewritten, by introducing the operators

$$
\left\{\begin{array}{l}
A_{\theta}=\cos (\theta) a+i \sin (\theta) a^{\dagger}, \\
B_{\theta}=\cos (\theta) a^{\dagger}+i \sin (\theta) a,
\end{array}\right.
$$

as

$$
H_{\theta}=\omega_{\theta}\left(B_{\theta} A_{\theta}+\frac{1}{2} \mathbb{1}\right)
$$

where $\omega_{\theta}=\frac{1}{\cos (2 \theta)}$ is well defined since $\cos (2 \theta) \neq 0$ for all $\theta \in I$. It is clear that $A_{\theta}^{\dagger} \neq B_{\theta}$ and that $\left[A_{\theta}, B_{\theta}\right]=\mathbb{1}$. In [6] we have proven that these operators satisfy Assumptions 1, 2 and 3 but not Assumption 4. In particular we have deduced that $\hat{S}_{\varphi}=|\alpha|^{2} e^{i \theta\left(a^{2}-a^{\dagger^{2}}\right)}$, where $\alpha \in \mathbb{C}$ is arbitrary but fixed. which is unbounded with unbounded inverse. Again, we find PB which are not regular. 


\section{Conclusions}

In this paper we have discussed the relation between RPB and PB with ordinary bosons. As the two theorems proven here clearly show, there is a strong connection between these excitations, at least under suitable assumptions. Which are the relevant assumptions are clarified by the theorems: for instance, if we just consider operators satisfying $[a, b]=\mathbb{1}$, this is not enough to

get any relevant functional structure. If, as an example, we take $a=\frac{d}{d x}, b=x$ and $\mathcal{H}=\mathcal{L}^{2}(\mathbb{R})$, no square integrable function $\varphi_{0}(x)$ exists with the required properties. So Assumption 1 (and Assumption 2 as well) is not satisfied. So we cannot introduce, starting from $a$ and $b$, a basis of $\mathcal{H}$. This suggests that, while Assumption 4 can be avoided, and Assumption 3 could be weakened by considering relevant subspaces of $\mathcal{H}$, Assumption 1 and 2 are absolutely necessary.

Further analysis on these operators are in progress.

\section{Acknowledgements}

The author acknowledge M.I.U.R. for financial support.

\section{References}

[1] F. Bagarello, Pseudo-bosons, Riesz bases and coherent states, J. Math. Phys., 50, 023531 (2010) (10pg)

[2] F. Bagarello Construction of pseudo-bosons systems, J. Math. Phys., 51, 053508 (2010) (10pg)

[3] F. Bagarello Mathematical aspects of intertwining operators: the role of Riesz bases, J. Phys. A, 43, 175203 (2010) (12pp)

[4] F. Bagarello, F. Calabrese Pseudo-bosons arising from Riesz bases, Bollettino del Dipartimento di Metodi e Modelli Matematici, 2, 15-26, (2010)

[5] D.A. Trifonov, Pseudo-boson coherent and Fock states, quant-ph/0902.3744

[6] F. Bagarello, Examples of Pseudo-bosons in quantum mechanics, Phys. Lett. A, 374, 3823$3827(2010)$ 
[7] S.T. Ali, F. Bagarello, J.-P. Gazeau, Modified Landau levels, damped harmonic oscillator and two-dimensional pseudo-bosons, J. Math. Phys., submitted

[8] J. Govaerts, C. M. Bwayi, O. Mattelaer, The KlauderDaubechies construction of the phasespace path integral and the harmonic oscillator, J. Phys. A, 42, 445304 (2009) (20pp)

[9] C. Bender, Making Sense of Non-Hermitian Hamiltonians, Rep. Progr. Phys., 70, 947-1018 (2007)

[10] A. Mostafazadeh, Pseudo-hermitian quantum mechanics, quant-ph/0810.5643

[11] A. Mostafazadeh, Conceptual aspects of PT-symmetry and pseudo-hermiticity: a status report, quant-ph/1008.4680

[12] Young R., An introduction to nonharmonic Fourier series, Academic Pree, New York, (1980)

[13] F. Bagarello, Pseudo-bosons, so far, Rev. Math. Phys., submitted

[14] Kuru S., Tegmen A., Vercin A., Intertwined isospectral potentials in an arbitrary dimension, J. Math. Phys, 42, No. 8, 3344-3360, (2001); Kuru S., Demircioglu B., Onder M., Vercin A., Two families of superintegrable and isospectral potentials in two dimensions, J. Math. Phys, 43, No. 5, 2133-2150, (2002); Samani K. A., Zarei M., Intertwined hamiltonians in two-dimensional curved spaces, Ann. of Phys., 316, 466-482, (2005).

[15] F. Bagarello Extended SUSY quantum mechanics, intertwining operators and coherent states, Phys. Lett. A, DOI: 10.1016/ j.physleta. 2008.08.047 (2008), F. Bagarello Vector coherent states and intertwining operators, J. Phys. A., doi:10.1088/1751-8113/42/7/075302, (2009), F. Bagarello, Intertwining operators between different Hilbert spaces: connection with frames, J. Math. Phys., DOI: 10.1063/1.3094758, 50, 043509 (2009) (13pp)

[16] S.T. Ali, J-P. Antoine and J-P. Gazeau, Coherent States, Wavelets and Their Generalizations, Springer-Verlag, New York, 2000.

[17] J-P. Gazeau, Coherent states in quantum physics, Wiley-VCH, Berlin 2009

[18] Christensen O., An Introduction to Frames and Riesz Bases, Birkhäuser, Boston, (2003)

[19] G. K. Pedersen, Analysis now, Springer-Verlag, New York 1989 
[20] V. L. Hansen, Functional analysis: entering Hilbert space, World Scientific, Singapore (2006)

[21] J. da Providência, N. Bebiano, J.P. da Providência, Non hermitian operators with real spectrum in quantum mechanics, arXiv:0909.3054 [quant-ph] 